\title{
Editorial: 2015 in Review
}

\author{
Fred R. Volkmar ${ }^{1}$
}

Published online: 18 February 2016

(c) Springer Science+Business Media New York 2016

Continuing a trend of more than 8 years, the number of papers submitted to the Journal continues to increase steadily, from 740 submissions in 2012, to 822 in 2013, to 940 in 2014 and nearly 900 this past year; the slightly smaller number this year reflects the fact that we're now redirecting review papers to the Review Journal of Autism and Developmental Disorders. Of the papers submitted last about $28 \%$ were ultimately accepted, though, in most cases, with one or more revisions. During the past year, the number of Associate Editors and Editorial Board members has been increased to manage the burgeoning paper volume more effectively and ensure that $J A D D$ maintains the highest quality for articles that are ultimately published.

The Journal also continues to strive to maintain an efficient turnaround time. During 2015, the average review time for the majority of papers-between first submission and initial decision-was about 40 days. Not surprisingly, given the increased volume of papers, the rejection rate continues to rise. We hope that the feedback provided to authors is helpful as they consider submission elsewhere. In addition, to conserve valuable Journal space, authors are encouraged to use digital resources to publish extra supplementary, but still valuable, materials that can reasonably be included in online appendices. We are grateful to the production staff at Springer for helping move submissions through the publication process so that now papers, once accepted, are published online in relatively short order (i.e., typically less than 2 weeks).

As always, I'm deeply grateful to the Associate Editors: Joshua Diehl, MD.; Christopher McDougle, Ph.D.; Carla

Fred R. Volkmar

lori.klein@yale.edu

1 New Haven, CT, USA
Mazefsky, Ph.D.; Ann S. Le Couteur, B.Sc., MBBS, FRCPsych, FRCPCH; James McPartland, Ph.D.; Nancy Minshew, MD; Brian Reichow, Ph.D.; and Jeremy M. Veenstra-Vanderweele, MD. Each has generously and indefatigably supported the work of the Journal, especially as the number of submissions continues to climb. During the past year, we have also welcomed the following new Associate Editor, Alice Carter, Ph.D., as we bid farewell to Sandra Harris, Ph.D., who served with great diligence as an associate editor for many years.

In the Journal office, Lori Klein, Editorial Manager, and our new Editorial Assistant, Logan Hart, work meticulously to ensure that papers conform to Journal standards before they enter the submission system, thereby enabling the Editor, Associate Editors, and Reviewers to more efficiently conduct the peer-review process. We also wish to thank Isaac Smith for his service this past year as the Editorial Assistant and wish him well in graduate school. And thanks to Judy Jones and the rest of the individuals at Springer who are involved in the production of the Journal, including Michelle Tam, Suganya Manikandan, and Koyal Bhattarcharjee.

We welcome the following new members to the Editorial Board, all very worthy having submitted several reviews this past year: Benjamin Yerys, Ph.D., from Children's Hospital of Philadelphia, and Connor Kerns, Ph.D., from Drexel University.

We also want to acknowledge the "Top 10" reviewers (i.e., those individuals who provided the greatest number of comprehensive, thorough, timely reviews) for the Journal this year:

1. Kenneth Aitken

2. Steve Kroupa

3. Tristram Smith

4. Christine Syriopoulou 
5. Giacomo Vivanti

6. Inge-Marie Eigst

7. Johnny Matson

8. Dorit ben Shalom

9. Lee Marcus

10. David Beversdorf
We list below the names of all individuals who have contributed one or more reviews to the Journal this year. Although far from perfect, peer review is the most effective way we have, as a field, to ensure the quality of our scholarly product. We thank all these individuals for their hard work and dedication.

\author{
A. Alexander \\ Aaron Kaat \\ Aaron Shield \\ Adam Feinstein \\ Ahmad Gharaibeh \\ Aja Murray \\ Akio Wakabayashi \\ Alan Brown \\ Alan Yu \\ Alanna Gold \\ Alastair Clarkson \\ Albert Cotugno \\ Alessandro Crippa \\ Alfred Chavira \\ Alice Carter \\ Alison Betz \\ Alison Davies \\ Alison Pilnick \\ Alison Presmanes Hill \\ Alison Singer \\ Allison Jack \\ Allison Nahmias \\ Allison Ratto \\ Allison Wainer \\ Alok Amraotkar \\ Alokananda Rudra \\ Alvi Azad \\ Alyssa Milot \\ Alyssa Orinstein \\ Alyssa Sawyer \\ Alyssa Schlenz \\ Amanda Bakian \\ Amanda Gulsrud \\ Amanda Richdale \\ Amanda Steiner \\ Ami Klin \\ Amie Duncan \\ Amy Azano \\ Amy Daniels \\ Amy Esler \\ Amy Fritsch \\ Amy Pearson \\ Amy Spriggs \\ Anastasia Kyvelidou
}

Anat Zaidman-Zait

André Diedrich

Andrea McDuffie

Andrea Samson

Andreas Eckert

Andrew Stanfield

Andrew Zimmerman

Andrew Whitehouse

Andy Shih

Anett Kaale

Angel Fettig

Angela Scarpa

Anjali Bhatara

Anjana Bhat

Anke Scheeren

Ann Lagges

Ann Le Couteur

Ann Mulder

Ann Neumeyer

Ann Sam

Anna Egan

Anna Lambrechts

Anna Ogliari

Anna Robinson

Anne Snow

Anne Wheeler

Anneke Louwerse

Anneli Kylliainen

Annelies de Bildt

Annette Estes

Annette Joosten

Annette Karmiloff-Smith

Anoek Oerlemans

Antoinette Sabatino DiCriscio

Antonio Narzisi

Antonio Hardan

Aparna Nadig

Aphroditi Gouvousis

Ardail Henry

Armando Bertone

Ashleigh Hillier

Ashley de Marchena

Aske Plaat

Atsushi Senju
Aubyn Stahmer

Audrey Smerbeck

Audrey Thurm

Audrey Blakeley-Smith

Ayumi Umino

Azadeh Kushki

Barbara Braddock

Barbara Saunders

Barbara Trimarco

Bart Boets

Behzad Khorashad

Ben Alderson-Day

Ben Ambridge

Ben Popple

Benjamin Thomas

Benjamin Yerys

Benjamin Zablotsky

Bernard Crespi

Bertram Ploog

Beth Glasberg

Beth Johnson

Beth Lanning

Beth Malow

Beth Pfeiffer

Betholyn Gentry

Bibi Huskens

Bien Lai

Blake Hansen

Blythe Corbett

Bojana Kuzmanovic

Bonita (Bonnie) Klein-Tasman

Bradley Davitt

Brandon Keehn

Breanne Byiers

Brenda Clark

Brenna Maddox

Brent Kelsen

Brent Vander Wyk

Brian Barger

Brian Boyd

Brian Lee

Brian Reichow

Brian Willoughby

Brianna Lewis 
Brittany Travers

Brooke Ingersoll

Bruce Stevenson

Bruce Tonge

Bryan Reimer

Bryna Siegel

Caitlin Conner

Calum Hartley

Camilla McMahon

Canan sola özgüç

Cara Cashon

Cara Pugliese

Carina de Klerk

Carissa Cascio

Carla Mazefsky

Carlisle Runge

Carlos Carona

Carmen Feldhaus

Carol Curtin

Carol Rockhill

Carol Schall

Carol Weitzman

Carolien Rieffe

Caroline Brown

Caroline Moul

Caroline Schwartz

Carolyn Birdgemohan

Carolyn Shivers

Carrie Allison

Carrie Ballantyne

Carrie Childs

Cary Stacy Smith

Catherine Best

Catherine Jones

Catherine Lord

Catherine Manning

Catherine Stamoulis

Cathleen Piazza

Cecilia Feeley

Cédric Galéra

Celia Tam

Celine Saulnier

Chadi Calarge

Chang-Jiang Yang

Charles Henry

Charline Urbain

Charlotte Montgomery

Chata Dickson

Cheong Sng

Cherie Green

Cheryl Dissanayake

Cheryl Klaiman

Cheryl Nye
Cheryl Sandford

Cheryl Tierney

Cheryl Wright

Cheyenne Laue

Chieko Kanai

Chien-Yu Pan

Ching-fen Hsu

Ching-lin Wu

Chris Ashwin

Chris Keary

Christin McDonald

Christina Schmitz

Christine Nordahl

Christine Reeve

Christine Syriopoulou

Christopher Baglio

Christopher Jarrold

Christopher Lemons

Christopher Manente

Christopher McDougle

Christopher Sharpley

Christopher Tullis

Claire Dente

Claire Dumont

Claire Vallotton

Clare Harrop

Clarisse Garriot Chatel

Claudia Hilton

Claudio Longobardi

Colby Chlebowski

Colin Campbell

Colin Palmer

Connie Kasari

Connie Sung

Connie Wong

Connor Kerns

Coral Kemp

Coralie Chevallier

Cory Shulman

Costel Darie

Courtney Burnette

Craig Erickson

Cristan Farmer

C.A. Pop nee Costescu

Cynthia Riccio

Cynthia Johnson

Cynthia Waugh

Cyrielle Derguy

D.J. Greene

Daisy Loyd

Damian Santomauro

Dan Coulter

Dana Jorgensen
Daniel Bagner

Daniel Bone

Daniel Coury

Daniel Hubbard

Daniel Maggin

Daniel Messinger

Daniel Molina

Daniel Moreno-De-Luca

Daniel Shumer

Daniel Tedesco

Daniel Yang

Daniel Meechan

Daniela Plesa

Darlene Groomes

Darren Hedley

David Badcock

David Berry

David Beversdorf

David Giles

David Simmons

David Skuse

David Trembath

David Williams

Dawn Sandt

Deanna Swain

Deborah Fein

Deborah Hales

Deborah Riby

Debra Cote

Debra Prykanowski

Denis Sukhodolsky

Denise Berte

Dermot M. Bowler

Devin Casenhiser

Diana Robins

Diana Tan

Diana Wasserman

Diane Williams

Dina Dajani

Domenic Cicchetti

Donald Rojas

Donald Oswald

Donna Murray

Dorit ben Shalom

Dorit Kliemann

Dorothea Floris

Dorothea Iannuzzi

Dorothy Stubbe

Doug VanderLaan

Ed Ritvo

Edina Kishonthy

Edith Bavin

Edward Brodkin 
Edwin Cook, Jr.

Eileen Crehan

Eileen Haebig

Eileen Simon

Einar Ingvarsson

Elena Grigorenko

Eli Lebowitz

Elina Jokiranta

Elisabeth Whyte

Elizabeth Biggs

Elizabeth Cridland

Elizabeth Getzel

Elizabeth Kelley

Elizabeth Kim

Elizabeth Lanter

Elizabeth Laugeson

Elizabeth Lawson

Elizabeth Milne

Elizabeth Pellicano

Elizabeth Redcay

Elizabeth Simmons

Elizabeth Starr

Ellahe Chabani

Ellen Leibenluft

Eman Alsayed

Emily Bouck

Emily Bremer

Emily Coderre

Emily Farran

Emily Gardiner

Emily Myers

Emily Neuhaus

Emily Simonoff

Emma Gowen

En-ho Yoon

Eric Bieniek

Eric Duku

Eric Hazen

Eric Olofson

Eric Storch

Eric Zander

Erica Hinckson

Ericka Wodka

Erika Ota

Erin Barton

Erin Bigler

Ernst VanBergeijk

Eron Friedlander

Ervin Poljac

Esperanza Gómez Durán

Eugenia Hernandez-Ruiz

Eva Hesselmark

Eva Loth
Evdokia Anagnostou

Eve LeBarton

Eve-Marie Quintin

Fabienne Naber

Farah El Zein

Fay Fletcher

Fernanda Fernandes

Fiona Knott

Fiona Miller

Franc Donkers

Francis Konstantinos

Frank Sansosti

Frederick Frankel

Frederick Shic

Fung Suk-Chun

G. Glickman

Gabriel Dichter

Gabriela Rosenblau

Gabrielle Carlson

Gabrielle Lee

Gael Orsmond

Gagan Joshi

Ganapathy Umaiorubagam

Garland Jones

Gary Mesibov

Gazi Azad

Geoffrey Bird

Geoffrey Hall

George Anderson

Gerald Goldstein

Gerrit Van Schalkwyk

Giacomo Vivanti

Gillian Baird

Gillian Erhabor

Gillian Stanley

Ginger Milne

Giorgia Picci

Giulia Righi

Gordon Ramsay

Grace Gengoux

Grace Iarocci

Greg Wallace

Gregory Barnes

Gregory Chasson

Gregory Light

Gregory Thorkelson

Gretchen Carlisle

Guiomar Oliveira

H. Gardener

Hanna Alonim

Hanna Cygan

Hanna Kovshoff

Hannah Schertz
Hannelore Ehrenreich

Hans Bogte

Hans-leo Teulings

Hasan Zaghlawan

Hawon Lee

Heath Matheson

Heather Brown

Heather Henderson

Heather Kuhaneck

Heather Nuske

Heidi Abadeh

Helen Marwick

Helen Tager-Flusberg

Helena Kraemer

Helena Rutherford

Henry Roane

Herbert Roeyers

Hideo Honda

Hilary Gardner

Hilde Geurts

Hillary Bush

Hoffmann Thomas

Hojjat Allah Haghgoo

Hsin-Yi Liang

Hwan Cui Koh

Iain McClure

Igor Burstyn

Iliana Magiati

Imad Zaheer

Inge Kamp-Becker

Inge-Marie Eigsti

Ira Cohen

James McPartland

Jeffrey Karst

Jeffrey Wood

Jenna Lequia

Jennifer Symon

Jennifer Beighley

Jennifer Elder

Jennifer Gillis

Jennifer Mayer

Jennifer McFarland-Whisman

Jennifer Musolff

Jennifer Foss-Feig

Jennifer Ledford

Jenny Downs

Jenny Fairthorne

Jeremy Jewell

Jeremy Turk

Jeremy Veenstra- Vanderweele

Jeroen Dewinter

Jessica A. Brian

Jessica Abrams 
Jessica Lester

Jessica Paynter

Jessica Waxler

Jewel Crasta

Jill Fodstad

Jill Harris

Jill Hollway

Jill Locke

Jill Shuster

Jim Bodfish

Jina Jang

Jin-Ding Lin

Jo Van Herwegen

Joan Peskin

Joanna Lomas Mevers

Jodi Duke

Johanna Lake

Johanna Rendle-Short

Johannes Rojahn

John Cannell

John Constantino

John Gargus

John Herrington

John McGrew

John Richey

John W. Harrington

Johnna Swartz

Johnny Matson

Jon Brock

Jonathan Campbell

Jonathan Delafield-Butt

Jonathan Tarbox

Jonathan Weiss

Joseph Lambert

Joseph Lucyshyn

Joseph Maes

Joseph P. McCleery

Joseph Snow

Joshua Diehl

Joshua Green

Joshua Roffman

Joshua Skewes

Joy Xin

Juan Carlos Gomez

Judah Koller

Judy Reaven

Julia Fisher

Julia Parish-Morris

Julie Crittendon

Julie Rubin

Julie Taylor

Julie Wolf

Justin Hampton
Justin Leaf

Justin Rowberry

Kaitlyn Wilson

Kale Edmiston

Kami Koldewyn

Kara Hume

Kara Styck

Karen Bearss

Karen Eskow

Karen Horridge

Karen Kuhlthau

Karen Tang

Karin Strid

Karla Schneider

Katarzyna Chawarska

Kate Fiske

Kate Sofronoff

Kate Tchanturia

Kate Woodcock

Katharina Krämer

Katharine Zuckerman

Katherine Belardi

Katherine Crea

Katherine Gotham

Katherine Stavropoulos

Katherine Walton

Kathleen A. Quill

Kathleen Hopf

Kathleen Rogers

Kathleen Zanolli

Kathryn McCabe

Kathy Koenig

Katie Denman

Kayla Ten Eycke

Keely Cheslack-Postava

Keith Allen

Keith Radley

Keith Shafritz

Kelly Blankenship

Kelly Price

Kelly Sheperd

Kelly Tanner

Kelly Whalon

Kenneth Aitken

Kenneth Gadow

Kent Williams

Kerri Nowell

Kerri Staples

Keven Lee

Kevin Antshel

Kevin Stephenson

Kim Dean

Kim Fournier
Kimberly Crosland

Kimberly Maich

Kimberly Murza

Kimberly Zlomke

Kirsten Schohl

Kirstin Birtwell

Klaus Libertus

Kris Evers

Kristall Day

Kristen Bottema-Beutel

Kristen Gillespie-Lynch

Kristen Lindgren

Kristen Linton

Kristen Powers

Kristie Asaro Saddler

Kristine Kulage

Kristy Dykshoorn

Kwang-Sun Blair

Kyle Bennett

Kyle Williams

L. Jarrett Barnhill

Laci Watkins

Lara Harris

Laraine McDonough

Larry Welkowitz

Laszlo Erdodi

Laura Anderson

Laura Crane

Laura D'Antuono

Laura Graham Holmes

Laura Guli

Laura Gutermuth Anthony

Laura Nota

Laura O. Smith

Laura Politte

Laura Schreibman

Lauren Bishop-Fitzpatrick

Lauren Goodwin

Lauren Herlihy

Lauren Kenworthy

Lauren Marsh

Lauren Schmitt

Lauren Taylor

Lauren Turner-Brown

Lauren McGrath

Laurent Mottron

Laurie Batchelder

Laurie Jeans

Laurie Sperry

Laurie Wellner

Lawrence Fung

Lawrence Scahill

Leann Smith 
Lee Marcus

Leif Ekblad

Lena McCue

Leonard Abbeduto

Leslie Rescorla

Letitia Naigles

Letizia Palumbo

Leyla Akoury Dirani

Lidia Gabis

Lien Van Eylen

Linda Bandini

Linda Caterino

Linda Graham

Linda Kelly

Linda LeBlanc

Linda Murdock

Linda O'Neill

Linda Pring

Linda Quirmbach

Linda Watson

Lindee Morgan

Lindsey O’Dell

Li-Nien Chien

Lisa Christensen

Lisa Croen

Lisa Henderson

Lisa Honigfeld

Lisa Nowinski

Lisa Turner

Lisa Wiggins

Logan Hart

Loisa Bennetto

Lonnie Zwaigenbaum

Lorena Fernández de la Cruz

Lorene Amet

Lori Briel

Lori-Ann Sacrey

Lorna Goddard

Lorna Timmerman

Lorraine Bahrick

Louisa Miller

Louise Kaczmarek

Luc Lecavalier

Lucie Bouvet

Lucy Bilaver

Lucy Foulkes

Luis Jimenez

Luke Curtis

Luther Kalb

Lynn Adams

Lynn Koegel

Lynn Waterhouse

M.C. Lai
M.D. Rutherford

Madeleine Grigg-Damberger

Madeline Harms

Maithilee Kunda

Maj-Britt Posserud

Mara Parellada

Marc Lanovaz

Marc Woodbury-Smith

Marco Garzitto

Marco Pino

Mareike Altgassen

Marek Chawarski

Maretha De Jonge

Margaret Flores

Margaret Semrud-Clikeman

Maria Acosta

Maria Casagrande

Maria Efstratopoulou

Maria Katsifaraki

Maria Mody

María Morales-Suárez-Varela

Maria Timberlake

Maria Valdovinos

Maria Wynne

Marianne Barton

Marie-Christine Potvin

Marieke de Vries

Marie-Pierre Baron

Marisa Filipe

Marisela Huerta

Marissa Diener

Marita Falkmer

Marji Erickson Warfield

Mark Jaime

Mark Nielsen

Mark Stokes

Mark Strauss

Mark Vida

Marsha Mailick

Marshalyn Yeargin-Allsopp

Martin Plawecki

Mary Douglas

Mary E. Van Bourgondien

Mary Hanley

Mary McHale-Molenda

Mary Stewart

Masahiro Hirai

Matia Finn-Stevenson

Matt Tincani

Matthew Albrecht

Matthew Belmonte

Matthew Bennett

Matthew Brock
Matthew Cranwell

Matthew Goodwin

Matthew Hollocks

Matthew Hudson

Matthew Lerner

Matthew Maenner

Matthew Mosconi

Matthew Segall

Matthew Siegel

Matthew Smith

Maude Schneider

Maureen Durkin

Maureen Kenny

Meagan Talbott

Meena Khowaja

Megan Boyle

Megan Foley Nicpon

Megan Griffin

Megan Tudor

Megan Freeth

Meghan Burke

Meghan Davidson

Meghan Miller

Melanie Pellecchia

Melaura Tomaino

Melinda Vanya

Melissa King

Melissa Kirkovski

Melissa Nosik

Merina Shrestha

Mette Elmose Andersen

Micah Mazurek

Michael Alessandri

Michael D. Powers

Michael Fitzgerald

Michael Sorter

Michael Bach

Michael Bloch

Michael English

Michael Ganz

Michael Morrier

Michael Siller

Michelle Curran

Michelle Dawson

Michelle Patriquin

Mieke Ketelaars

Miho Kuroda

Mikhail Kissine

Mikle South

Miklos Gyori

Ming Wai Wan

Miriam Rosenberg-Lee

Mirko Uljarevic 
Misa Kayama

Missy Teatero

Mitzi Waltz

Miyuki Torii

Mohamed Bencherif

Mohammad Ghaziuddin

Mohammed Alzyoudi

Motomi Toichi

Moya Kinnealey

Muhammad Azeem

Muideen Bakare

Myra Beth Bundy

N. Russo-Ponsaran

Nalin Payakachat

Nancy Cheak-Zamora

Nancy Garon

Nancy Moss-Rascusin

Naomi Davis

Natália Correia

Natalia Kleinhans

Natalie Russo

Natalie Webb

Natascia Brondino

Natasha Marrus

Nathaniel Laor

Neill Broderick

Nicholas Chown

Nicholas Gelbar

Nicholas Riches

Nicole Baumer

Nicole Kreiser

Nicole Matthews

Nicole Turygin

Nicole Zurcher

Nidal Daou

Nigel Newbutt

Nikki Kiyimba

Niko Kargas

Nirit Bauminger-Zviely

Noah Sasson

Noha Minshawi

Nouchine Hadjikhani

Nuri Reyes

Olga Solomon

Olive Healy

Olivia Veatch

Omri Weisman

Opal Ousley

Oren Shtayermman

Ovsanna Leyfer

Oyku Mance Calisir

P. Krakowiak

Paige Cervantes
Paige Weinger

Pamela Bryden

Pamela Crooke

Pamela Pallett

Pamela Ventola

Panagiotis Siaperas

Paola Venuti

Pasquale Accardo

Patricia Howlin

Patricia Kloosterman

Patricia Lockwood

Patricia Manning-Courtney

Patricia Rao

Patricia van Wijngaarden- Cremers

Patrick Powell

Paul Ashwood

Paul Constable

Paul Gringras

Paul J. Yoder

Paul R. Benson

Paul Shawler

Paul Stevens

Paul Wang

Paula Goldenberg

Pauline Filipek

Pawan Sinha

Peng Zhou

Per Andersen

Peter Carpenter

Peter Chung

Peter Doehring

Peter Enticott

Peter Marschik

Peter Mundy

Peter Rabins

Peter Tanguay

Peter W. Dowrick

Phil Reed

Piyapa Keawutan

Priscilla Dseagu

Quentin Guillon

R. Douglass Greer

R.A. Mason

R.C. Sheldrick

Raanan Raz

Rachael Bedford

Rachel Aaron

Rachel Cole-Fletcher

Rachel Giora

Rachel Goldin

Rachel Grove

Rachel Jellett

Rachel Loftin
Rachna Sehgal

Rafal Zielinski

Rajesh Kana

Rajneesh Mahajan

Ralf Tepest

Ramesh Kaipa

Ramesh Rajan

Ramona Simut

Rano Engstrand

Raphae Bernier

Rebecca Chamberlain

Rebecca Edmondson Pretzel

Rebecca Elias

Rebecca Hardiman

Rebecca Landa

Rebecca Lawson

Rebecca Lucas

Renae Beaumont

Rene Jamison

Rhea Paul

Rhiannon Luyster

Richard Fleming

Richard Jolley

Rinat Gold

Rini Vohra

Risto Ilmoniemi

Rita Eagle

Riva Ritvo

Roald Oien

Rob van Lier

Robert Drake

Robert Hodapp

Robert Koegel

Robert LaRue

Robert Rada

Robert Rieske

Robie Schriber

Robin Gabriels

Robyn Young

Roger Jou

Rolanda Maxim Gott

Ron Thibert

Ronald Leaf

Ronna Fried

Rory Allen

Rosaria Furlano

Rose Nevill

Roxann Roberson-Nay

Roy Brown

Rubin Jure

Rupa Gordon

Rutger van der Gaag

Ruth Brenner 
Ruth Gerson

Ruth Grossman

Ruth Irene Eren

Ruth Van der Hallen

Ryan Adams

Ryan Stevenson

Salih Rakap

Sally J. Rogers

Sally Ozonoff

Samuel Odom

Samy Azer

Sana Abu-Dahab

Sandra Costa

Sandra Harris

Sandra Maestro

Sandra Maria Herondina Coelho

Aguiar

Sandra Tsang

Sanja Banjavcic

Sara Kover

Sara Spencer

Sarabeth Broder-Fingert

Sarah Allen

Sarah Carrington

Sarah De Pauw

Sarah Frampton

Sarah Haigh

Sarah Hampton

Sarah Hurwitz

Sarah Landsberger

Sarah Paterson

Sarah Pelangka

Sarah Schipul

Sarah Schoen

Sarah Spence

Sarah White

Sarah Wigham

Saray Bonete

Sarika Peters

Sasha Zeedyk

Sayyed Ali Samadi

Scott Jackson

Sebastian Gaigg

Selda Ozdemir

Selin Aktan Guloksuz

Sergio Melogno

Shannon Wagner

Sharon Jones

Sharon Kidd

Sharon McLaughlin

Sharon Reeve

Shaun Eack

Shawn Christ
Sheila Christopher

Sheryl Gettings

Shirli Wener

Shivani Sharma

Shota Uono

Shuo Wang

Sibel Kazak Berument

Sigal Eilat-Adar

Sigmund Eldevik

Silvia De Rubeis

Silvio Loddo

Simmie Foster

Simon Baron Cohen

Simon Gregory

Sloane Burgess

Smadar Dolev

So Hyun Kim

Sofia Mavropoulou

Soichiro Matsuda

Soile Loukusa

Sonia Martínez-Sanchis

Sophie Molholm

Spencer Hayes

Stacey Reynolds

Stacy Shumway

Stefanie Bodison

Stelios Georgiades

Stephanie Ameis

Stephanie Bader

Stephanie Bryson

Stephanie Hayes

Stephanie Shire

Stephen Benning

Stephen Edward McMillin

Stephen Kanne

Stephen Sheinkopf

Steve Grossberg

Steve Kroupa

Steven Chesnut

Sue Fletcher-Watson

Sunny Kim

Susan Campbell

Susan Faja

Susan Hedges

Susan Hepburn

Susan Hyman

Susan Jarmuz-Smith

Susan Jones

Susan Klein

Susan Malcolm-Smith

Susan Peppe

Susan Perlman

Susan Schultz
Susan White

Susana Mouga

Sushie Dobbinson

Susumu Yokota

Suzanne Macari

Sven Bolte

Swati Phatak

Sylvie Beauchamp

Sylvie Goldman

Takeshi Nishiyama

Tamara Daley

Tanja Sappok

Tatja Hirvikoski

Taylor Day

Teal Benevides

Ted Hutman

Telma Almeida

Teresa Bennett

Teresa May-Benson

Teresa Tavassoli

Terje Falck-Ytter

Terry Falcomata

Terry Overton

Tess Clifford

Tessa Taylor

Thayne Sweeten

Themba Carr

Theodore Tomeny

Thierry Chaminade

Thomas Berney

Thomas Fernandez

Thomas Frazier

Thomas L. Layton

Thomas Ollendick

Tia Schultz

Tiffany Woynaroski

Tina Goldsmith

Todd Levine

Tom Booth

Tom Loucas

Tony Charman

Treasa Murphy

Trine Lise Bakken

Tristram Smith

Ty Vernon

Tyler Hassenfeldt

Tze Jui Goh

Urvakhsh Mehta

Uttama Lahiri

Valentina Iemmi

Valérie Van Hees

Valsamma Eapen

Vanessa Bal 


\section{Vanessa Hus}

Vasiliki Totsika

Veronica Fleury

Vicki Bitsika

Victoria Hallett

Victoria Knight

Vikki Janke

Viktoria Lyons

Viktoriya Wormann

Vincent Billock

Vincent Guinchat

Virginie Cobigo
W.H. James

Wendy Klein

Wendy Blumenthal

William Grant

William Hudenko

William Mandy

William Sharp

Xenia Borue

Xin Tong

Xin Wei

Ya-Chih Chang

Yael Granader
Yael Kimhi

Yamini Howe

Yanki Yazgan

Yawei Cheng

Yoed Kenett

Yona Lunsky

Young She Bae

Yukari Takarae

Yvonne Groen

Zachary Warren 